\title{
Controlling Performance and Physiological Parameters for Body Size and Inter-individual Variability due to Biological Maturation during Adolescent Growth Spurt
}

\author{
Manuel J Coelho-e-Silva ${ }^{1 *}$, João Valente-dos-Santos ${ }^{1}$, João Duarte ${ }^{1}$, Dominika M Pindus ${ }^{2}$, Lauren B Sherar ${ }^{2}$ and Robert M Malina ${ }^{3,4}$ \\ ${ }^{1}$ Faculty of Sport Sciences and Physical Education, University of Coimbra, Coimbra, Portugal \\ ${ }^{2}$ School of Sport, Exercise and Health Sciences, Loughborough University, Loughborough, Leicestershire, UK \\ ${ }^{3}$ Department of Kinesiology and Health Education, University of Texas at Austin, USA \\ ${ }^{4}$ Department of Kinesiology, Tarleton State University, Stephenville, Texas, USA
}

\section{Introduction}

As individuals grow, size of the body (height and weight) and specific organs increase. Different segments of the body and components of body mass, however, vary in the tempo and timing of growth. For example, the brain and the head will have already reached approximately $50 \%$ the mature size by about two years of age, while mature size is usually attained between 8 and 10 years of age. In contrast, the reproductive organs are later in growth reaching only $10 \%$ (in absolute terms) of adult size by the ages of 10 to 12 years. Puberty is characterized by accelerated tempo of growth evident in the growth spurt in height and associated increases in body size and mass, and also in the growth of the reproductive organs.

Physiological functions are routinely expressed relative to stature or body mass. For example, peak oxygen uptake is commonly reported in millilitres per minute per unit body mass $\left(\mathrm{ml} \mathrm{kg}^{-1} \mathrm{~min}^{-1}\right)$. Fat-free mass, body surface area and stature are also used as denominators. However, issues related to the validity of estimates of fat free mass and body surface area need to be considered if these variables are used as denominators. For example, $54 \%$ and $52 \%$ of the variance in anaerobic mean and peak power obtained with the Wingate test (WAnT) was predicted by biological maturation, body mass and leg length in Portuguese adolescent basketball players aged 14-16 years. Biological maturation was expressed as the ratio of skeletal age (SA) to chronological age (CA) [1]. The WAnT performance is often reported in Watt per unit of body mass or per unit of thigh volume.

\section{Ratio standards, isometry and elastic similarity}

Simple ratio standards $\left(\mathrm{mL} \mathrm{kg}^{-1} \mathrm{~min}^{-1} ; \mathrm{W} \mathrm{kg}^{-1} ; \mathrm{W} \mathrm{L} \mathrm{L}^{-1}\right)$ have been strongly criticised [2,3]. Expressing functions relative to anthropometric dimensions is meant to control for inter-individual variability in body size and is based on the assumption of geometric similarity. The ratios rely on the assumption that variables expressed as the ratio are linearly related, but the assumption can be erroneous. Although ratio standards are commonly used to control for the effects of body size on performance, other methods provide plausible alternatives.

Relationships among length, surface area and volume have implications for metabolism and thermoregulation. All linear anthropometric dimensions of the body, such as stature, segment lengths, and breadths have the dimension $d$. All areas including body surface area and muscle cross-sectional areas have the dimension $d^{2}$. Total body volume given by air displacement plethysmography and other volumes (lung, heart, lower limb) have the dimension $d^{3}$. According to the second Newtonian law, time has the dimension $d^{1}$. Thus, peak oxygen uptake measured as volume per unit time $\left(\mathrm{L} \mathrm{min}^{-1}\right.$ ) should be proportional to $\mathrm{d}^{3}-\mathrm{d}^{1}$. In order to dissociate peak oxygen uptake from body size given by stature $(\mathrm{m})$, values should be expressed in $\mathrm{m}^{2}$. In isometric bodies stature corresponds to mass raised to $2 / 3$ power function (assumption of geometric similarity) and an alternative denominator for peak oxygen uptake would be $\mathrm{mL} \mathrm{kg} \mathrm{kg}^{-0.67} \mathrm{~min}^{-1}$. However, a scaling exponent of mass rose to the power 0.75 (stature ${ }^{2.25}$ ) has also been suggested. Observations suggest that metabolic rate in many species of homeotherms conforms to mass raised to the power of 0.75 and not to the expected surface law of mass raised to the power of 0.67 . A rationale for this apparent departure from theoretical predictions was offered by a model of elastic similarity [4]. This model using a 0.75 exponent was proposed in zoology, but has been questioned as a statistical artefact because it was based on a single allometric model applied to data for a number of different species. When the data from specific species was interpreted separately, it had an intra-specific exponent of 0.67 . These observations raise another question: how valid is the method of extrapolating an exponent for resting oxygen uptake in calculating maximal oxygen uptake?

\section{Assumptions for ratio standard, fundamentals of power functions and proportional allometric modelling}

Performance and physiological data often have a normal distribution. If the data have longer tail to the right side of the distribution, the data are described as positively skewed. In contrast, if the longer tail occurs on the left side of the distribution, the data are described as negatively skewed. Certain transformations can be used to overcome the problems of skewed data to correct the asymmetry and provide a normal distribution. For example, a $\log$ transformation will frequently correct positively skewed data such as body mass $(\mathrm{kg})$ or maximal oxygen uptake $\left(\mathrm{L} \mathrm{min}^{-1}\right)$ into a 'log-normal' distribution. The Shapiro-Wilk test (SPSS) indicates whether the data fit a normal distribution.

A ratio standard is valid only when the product-moment correlation coefficient between variables $\mathrm{X}$ and $\mathrm{Y}$ corresponds to coefficient of variation of $\mathrm{X}$ (standard deviation of $\mathrm{X}$ divided by the mean of X) divided by the coefficient of variation of $\mathrm{Y}$ (standard deviation of $\mathrm{X}$ divided by the mean of $\mathrm{X}$ ).

Allometric models present an alternative to ratio standards in assessing the effects of size-related increments on performance. An example of the allometric model is presented in eq. (1), where $a$ is the

*Corresponding author: Manuel J Coelho-e-Silva, Faculty of Sport Science and Physical Education, University of Coimbra, Portugal, E-mail: mjcesilva@hotmail.com Received October 18, 2013; Accepted October 19, 2013; Published October 26 2013

Citation: Coelho-e-Silva MJ, Valente-dos-Santos J, Duarte J, Pindus DM Sherar LB, et al. (2013) Controlling Performance and Physiological Parameters for Body Size and Inter-individual Variability due to Biological Maturation during Adolescent Growth Spurt. J Sports Med Doping Stud 3: e137. doi:10.4172/21610673.1000e137

Copyright: () 2013 Coelho-e-Silva MJ, et al. This is an open-access article distributed under the terms of the Creative Commons Attribution License, which permits unrestricted use, distribution, and reproduction in any medium, provided the original author and source are credited. 
Citation: Coelho-e-Silva MJ, Valente-dos-Santos J, Duarte J, Pindus DM, Sherar LB, et al. (2013) Controlling Performance and Physiological Parameters for Body Size and Inter-individual Variability due to Biological Maturation during Adolescent Growth Spurt. J Sports Med Doping Stud 3: e137. doi:10.4172/2161-0673.1000e137

Page 2 of 2

intercept of the regression line on the y-axis and $b$ is the slope of the line used to model the relationship between performance outputs and variables associated with body size, and $e$ is a multiplicative error term. Values of $a$ and $b$ are derived from linear regressions of the logarithmic regression transformations of eq. (1) that are given in eq. (2).

$$
\begin{aligned}
& \mathrm{Y}=\mathrm{aX}^{\mathrm{b}} \mathrm{e} \\
& \log (\mathrm{Y})=\log (\mathrm{a})+\mathrm{b} \times \log (\mathrm{X})+\log (\mathrm{e})
\end{aligned}
$$

Exponents for stature and thigh volume from the independent allometric models of peak $\mathrm{VO}_{2}$ derived from eq. (2) were determined for adolescent roller hockey players [5]. Exponents were 2.15 (95\%CI: 1.37 to 2.94 ) for stature and 0.55 (95\%CI: 0.41 to 0.69 ) for anthropometrically estimated thigh volume. The models for stature explained $30 \%$ and those for thigh volume explained $46 \%$ of the variance in peak $\mathrm{VO}_{2}$. The residuals of these simple allometric models were not correlated with the residuals of their respective body size variables. Visual inspection of absolute residuals showed no heteroscedasticity, indicating that stature and thigh volume can be used to derive peak $\mathrm{VO}_{2}$ independent of size. However, statistically significant medium sized correlations were apparent when residuals of the simple allometric models were correlated with another variable related to body size (e.g., residuals of peak $\mathrm{VO}_{2}$ modelled for thigh volume against stature) $(0.15<r<0.33)$. In other words, stature or thigh volume alone did not correctly partition out the influence of body size on peak $\mathrm{VO}_{2}$. Consequently, proportional allometric models were explored (eq. (3)). Multiple stepwise regressions were conducted using the linearized equation with logtransformations (eq. (4)) [6-8].

$$
\mathrm{Y}=\text { size descriptor } 1^{\mathrm{K} 1} \times \text { size descriptor } 2^{\mathrm{K} 2} \times \mathrm{e}
$$

$\log (\mathrm{Y})=\mathrm{K}_{1} \times \log ($ size descriptor 1$)+\mathrm{K}_{2} \times \log ($ size descriptor 2$)+$

$\log (\mathrm{e})$

Other models that incorporate CA or SA can also be explored (eq. (5)). Eq. (5) can be linearized with a log-transformation as in eq. (6).

$$
\begin{aligned}
& \mathrm{Y}=\text { size descriptor }^{\mathrm{K}} \times \exp (\mathrm{c}+\mathrm{d} \times \mathrm{CA} \text { or SA }) \times \mathrm{e} \\
& \log (\mathrm{Y})=k \log (\text { size descriptor })+c+d(\mathrm{CA} \text { or } \mathrm{SA})+\log \varepsilon
\end{aligned}
$$

In the study with male adolescent hockey players the models also incorporated $\mathrm{CA}$ and $\mathrm{CA}^{2}$ and $\mathrm{SA}$ and $\mathrm{SA}^{2}$ as exponential terms in addition to stature and thigh volume [5]. Eq. (6) corresponds to a candidate model that considered CA and SA as a quadratic polynomial to allow the nonlinear relationship with peak $\mathrm{VO}_{2}$.

\section{Conclusion}

Simple per body mass ratios (e.g. $\mathrm{mL} \mathrm{kg}^{-1} \mathrm{~min}^{-1}$ ) often remain size dependent thus confounding interpretations. Linear regression scaling is limited by its assumption of an additive error term and taking into account that most performance parameters are characterized by heteroscedastic (multiplicative) error terms, allometric (log-linear) scaling techniques facilitate the construction of appropriately size-adjusted ratio. Mass exponents close to or equal to 0.67 provided support for more general application of the $2 / 3$ power suggested by geometrically similarity, especially in studies modelling adult peak oxygen uptake. The application of allometry to longitudinal data is more complex. Ontogenetic allometry describes differential growth in the individual growth process but cannot quantify group developmental changes. When different individuals are measured at the same developmental stage within a population it is called a static allometry. These allometric procedures can be used to account for intra- and inter-individual variability, respectively. However, allometric multilevel modeling offers many advantages evaluating longitudinal changes as it allows simultaneous estimation of the within and among individuals variation.

\section{References}

1. Carvalho HM, Coelho-e-Silva MJ, Gonçalves CE, Philippaerts R, Castagna C, et al. (2011) Age-related variation of maximal short-term effort after controlling for size and maturation in adolescent athletes. Ann Hum Biol 38: 721-727.

2. Tanner JM (1949) Fallacy of per-weight and per-surface area standards and their relation to spurious correlation. J Appl Physiol 2: 1-15.

3. Katch VL, Katch FI (1974) Use of weight-adjusted oxygen uptake scores that avoid spurious correlation. Res Q 45: 447-451.

4. McMahon T (1973) Size and shape in biology: elastic criteria impose limits on biological proportions, and consequently on metabolic rates. Science: 1201-1204.

5. Valente-dos-Santos J, Sherar L, Coelho-e-Silva M, Pereira JR, Vaz V, et al. (2013) Allometric scaling of peak oxygen uptake in male roller hockey players under 17 years old. Appl Physiol Nutr Metab 38: 390-395.

6. Nevill AM, Holder RL, Baxter-Jones A, Round JM, Jones DA (1998) Modelling developmental changes in strength and aerobic power in children. J Appl Physiol 84: 963-970.

7. Welsman JR, Armstrong N (2008) Interpreting exercise performance data in relation to body size. In N Armstrong, W van Mechelen (Eds) Paediatric Exercise Science and Medicine. Oxford University Press, Oxford, 13-22.

8. Valente-dos-Santos J, Coelho-e-Silva MJ, Castanheira J, Ronque ER, Elferink-Gemser MT, et al. (2013) Modelling developmental changes in left ventricular mass using multiplicative allometric and additive polynomial multilevel modelling in boys aged 11-16 years. In MJ Coelho-e-Silva, A Cupido-dos-Santos, AJ Figueiredo, JP Ferreira, and N Armstrong (Eds) Children and Exercise XXVIII: The proceedings of the 28th Peadiatric Work Physiology Meeting. Oxon, UK: Routledge. 